\title{
SOME PROPOSITIONS EQUIVALENT TO THE CONTINUUM HYPOTHESIS
}

\author{
BY FREDERICK BAGEMIHL \\ Communicated by Deane Montgomery, December 11, 1958
}

Let $\mathcal{E}$ denote the real line. If $T \subset \mathcal{E}$ and $r \in \mathcal{E}$, we set $\{t+r: t \in T\}$ $=T[r]$. In [1] we have proved these two theorems:

$\left(\mathrm{B}_{\mathrm{K}}\right)$ Let $S \subset \mathcal{E}, T \subset \mathcal{E}, S$ be at most enumerable and $T$ be of first category. Then $\mathcal{E}$ contains a residual subset $R$ such that $S \cap T[r]$ is empty for every $r \in R$.

$\left(\mathrm{B}_{\mathrm{M}}\right)$ Let $S \subset \mathcal{E}, T \subset \mathcal{E}, S$ be at most enumerable and $T$ be of measure zero. Then $\mathcal{E}$ contains a subset $R$ such that $\mathcal{E}-R$ is of measure zero and $S \cap T[r]$ is empty for every $r \in R$.

We introduce the following propositions:

$\left(\mathfrak{B}_{\mathrm{K}}\right)$ Let $S \subset \mathcal{E}, T \subset \mathcal{E}, S$ be of power less than $2^{\mathrm{N}_{0}}$ and $T$ be of first category. Then $\&$ contains a residual subset $R$ such that $S \cap T[r]$ is empty for every $r \in R$.

$\left(\mathfrak{B}_{\mathrm{M}}\right)$ Let $S \subset \mathcal{E}, T \subset \mathcal{E}, S$ be of power less than $2^{\mathbb{N}_{0}}$ and $T$ be of measure zero. Then $\mathcal{E}$ contains a subset $R$ such that $\mathcal{E}-R$ is of measure zero and $S \cap T[r]$ is empty for every $r \in R$.

$\left(\mathfrak{B}_{K}^{*}\right)$ Let $S \subset \mathcal{E}, T \subset \mathcal{E}, S$ be of power less than $2^{\mathrm{N}_{0}}$ and $T$ be of first category. Then there exists an $r \in \mathcal{E}$ such that $S \cap T[r]$ is empty.

$\left(\mathfrak{B}_{M}^{*}\right)$ Let $S \subset \mathcal{E}, T \subset \mathcal{E}, S$ be of power less than $2^{\mathbb{N}_{0}}$ and $T$ be of measure zero. Then there exists an $r \in \mathcal{E}$ such that $S \cap T[r]$ is empty.

Clearly $\left(\mathfrak{B}_{\mathrm{K}}\right)$ implies $\left(\mathfrak{B}_{K}^{*}\right)$ and $\left(\mathfrak{B}_{\mathrm{M}}\right)$ implies $\left(\mathfrak{B}_{M}^{*}\right)$.

The following five propositions are discussed at some length in [2]:

(H) $2 \aleph_{0}=\aleph_{1}$.

$(\Omega)$ The union of less than $2^{N_{0}}$ subsets of $\&$ of first category is of first category.

$(\mathfrak{M})$ The union of less than $2^{\mathrm{N}_{0}}$ subsets of $\mathcal{E}$ of measure zero is of measure zero.

$\left(\Omega^{*}\right) \mathcal{E}$ is not the union of less than $2^{N_{0}}$ subsets of $\&$ of first category.

$\left(\mathfrak{M}^{*}\right) \mathcal{E}$ is not the union of less than $2^{\mathbb{N}_{0}}$ subsets of $\mathcal{E}$ of measure zero.

Evidently $(\mathrm{H})$ implies $(\Re)$ and $(\mathfrak{M}),(\Omega)$ implies $\left(\Omega^{*}\right)$, and $(\mathfrak{M})$ implies $\left(\mathfrak{M}^{*}\right)$.

By examining the proofs of $\left(B_{K}\right)$ and $\left(B_{M}\right)$, it is easy to see that the following lemma is true.

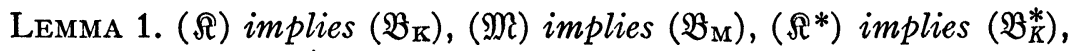
and $\left(\mathfrak{M}^{*}\right)$ implies $\left(\mathfrak{B}_{M}^{*}\right)$.

Now let $\odot$ denote the plane provided with a Cartesian coordinate 
system having a horizontal $x$-axis and a vertical $y$-axis. If $\Phi$ is a family of horizontal lines (in $\odot$ ), we say that $\Phi$ is of first category (measure zero) if the union of the members of $\Phi$ intersects the $y$-axis in a linear set of first category (measure zero). If $r \in \mathcal{E}$, we denote by $\Phi[r]$ the family of horizontal lines obtained from $\Phi$ as follows: if $L$ is a member of $\Phi$ and intersects the $y$-axis at $y_{0}$, then the horizontal line that intersects the $y$-axis at $y_{0}+r$ is made a member of $\Phi[r]$. We call the families $\Phi[r](r \in \mathcal{E})$ the translations of $\Phi$.

We introduce also the following propositions:

$\left(\mathfrak{Q}_{\mathrm{K}}\right)$ There exists a subset $A$ of $P$ and a family $\Phi$ of horizontal lines such that

(i) $\Phi$ is of first category,

(ii) there is a subset $U$ of $\mathcal{E}$ of second category such that, for every $u \in U$, the family $\Phi[u]$ contains a horizontal line that intersects $A$ in at most $\boldsymbol{\aleph}_{0}$ points,

(iii) every member of some nonenumerable set of vertical lines intersects $\mathbb{P}-A$ in at most $\boldsymbol{\aleph}_{0}$ points.

$\left(\mathfrak{Q}_{\mathrm{M}}\right)$ There exists a subset $A$ of $\odot$ and a family $\Phi$ of horizontal lines such that

(i) $\Phi$ is of measure zero,

(ii) there is a subset $U$ of $\mathcal{E}$ of positive exterior measure such that, for every $u \in U$, the family $\Phi[u]$ contains a horizontal line that intersects $A$ in at most $\aleph_{0}$ points,

(iii) every member of some nonenumerable set of vertical lines intersects $\boldsymbol{P}-A$ in at most $\aleph_{0}$ points.

$\left(\mathfrak{Q}_{K}^{*}\right)$ There exists a subset $A$ of $\odot$ and a family $\Phi$ of horizontal lines such that

(i) $\Phi$ is of first category,

(ii) every translation of $\Phi$ contains a horizontal line that intersects $A$ in at most $\aleph_{0}$ points,

(iii) every member of some nonenumerable set of vertical lines intersects $\boldsymbol{P}-A$ in at most $\aleph_{0}$ points.

$\left(\mathfrak{Q}_{M}^{*}\right)$ There exists a subset $A$ of $\odot$ and a family $\Phi$ of horizontal lines such that

(i) $\Phi$ is of measure zero,

(ii) every translation of $\Phi$ contains a horizontal line that intersects $A$ in at most $\boldsymbol{\aleph}_{0}$ points,

(iii) every member of some nonenumerable set of vertical lines intersects $\mathbb{P}-A$ in at most $\aleph_{0}$ points.

$\left(\mathfrak{Q}_{K}^{\prime}\right)$ There exists a subset $A$ of $\odot$ and a family $\Phi$ of horizontal lines such that

(i) $\Phi$ is of power less than $2 \aleph_{0}$, 
(ii) there is a subset $U$ of $\mathcal{E}$ of second category such that, for every $u \in U$, the family $\Phi[u]$ contains a horizontal line that intersects $A$ in at most $\boldsymbol{\aleph}_{0}$ points,

(iii) every member of some nonenumerable set of vertical lines intersects $\mathbb{P}-A$ in a linear set of first category.

$\left(\mathfrak{Q}_{M}^{\prime}\right)$ There exists a subset $A$ of $\odot$ and a family $\Phi$ of horizontal lines such that

(i) $\Phi$ is of power less than $2 \mathrm{~N}_{0}$,

(ii) there is a subset $U$ of $E$ of positive exterior measure such that, for every $u \in U$, the family $\Phi[u]$ contains a horizontal line that intersects $A$ in at most $\boldsymbol{\aleph}_{0}$ points,

(iii) every member of some nonenumerable set of vertical lines intersects $\beta-A$ in a linear set of measure zero.

Obviously $\left(\mathfrak{Q}_{K}^{*}\right)$ implies $\left(\mathfrak{Q}_{K}\right)$ and $\left(\mathfrak{Q}_{M}^{*}\right)$ implies $\left(\mathfrak{Q}_{M}\right)$. We remark that Propositions $(\mathfrak{B})$ and $(\mathfrak{B})$ in $[2]$ imply $\left(\mathfrak{Q}_{K}^{\prime}\right)$ and $\left(\mathfrak{Q}_{M}^{\prime}\right)$, respectively.

Lemma 2. (H) implies $\left(\mathfrak{Q}_{K}^{*}\right),\left(\mathfrak{Q}_{M}^{*}\right),\left(\mathfrak{Q}_{K}^{\prime}\right)$, and $\left(\mathfrak{Q}_{M}^{\prime}\right)$.

Proof. Suppose that $(\mathrm{H})$ is true. Then $\left[3\right.$, p. 9, Proposition $\left.P_{1}\right]$ there exists a subset $A$ of $\mathcal{P}$ such that the intersection of every horizontal line with $A$ is an at most enumerable set and the intersection of every vertical line with $P-A$ is an at most enumerable set; if we let $\Phi$ consist of a single horizontal line, the truth of $\left(\mathfrak{\Omega}_{K}^{*}\right)$, $\left(\mathfrak{Q}_{M}^{*}\right),\left(\mathfrak{\Omega}_{K}^{\prime}\right)$, and $\left(\mathfrak{Q}_{M}^{\prime}\right)$ is apparent.

Theorem 1. The conjunction of $\left(\mathfrak{B}_{\mathrm{K}}\right)$ and $\left(\mathfrak{Q}_{\mathrm{K}}\right)$ is equivalent to $(\mathrm{H})$.

Proof. (a) Assume that $(\mathrm{H})$ is true. Then Lemma 1 implies that $\left(\mathfrak{B}_{K}\right)$ is true, and the truth of $\left(\mathfrak{Q}_{K}\right)$ follows from Lemma 2.

(b) Assume that $\left(\mathfrak{B}_{\mathrm{K}}\right)$ and $\left(\mathfrak{Q}_{\mathrm{K}}\right)$ are true. If $(\mathrm{H})$ is false, then, in view of (iii) of $\left(\mathfrak{Q}_{\mathrm{K}}\right)$, there exist $\mathfrak{p}$ vertical lines, with $\boldsymbol{\aleph}_{0}<\mathfrak{p}<2 \boldsymbol{\aleph}_{0}$, whose union intersects $P-A$ in a set whose orthogonal projection, $S$, on the $y$-axis is of power less than $2^{N_{0}}$. If $T$ is the intersection of the $y$-axis with the union of the members of $\Phi$, then, by (i) of $\left(\mathfrak{Q}_{\mathrm{K}}\right), T$ is a linear set of first category, and $\left(\mathscr{B}_{\mathrm{K}}\right)$ implies that $\varepsilon$ contains a residual subset $R$ with the property that $S \cap T[r]$ is empty for every $r \in R$. This means that, for some $u \in U$, every member of $\Phi[u]$ intersects each of the aforementioned $\mathfrak{p}$ vertical lines in a point of $A$, which contradicts (ii) of $\left(\mathfrak{Q}_{\mathrm{K}}\right)$. Consequently, $(\mathrm{H})$ is true.

THEOREM 2. The conjunction of $\left(\mathfrak{B}_{\mathrm{M}}\right)$ and $\left(\mathfrak{Q}_{\mathrm{M}}\right)$ is equivalent to $(\mathrm{H})$.

Proof. In the proof of Theorem 1 , replace " $\left(\mathfrak{B}_{\mathrm{K}}\right)$ " by "( $\left.\mathfrak{B}_{\mathrm{M}}\right)$ ", 
"( $\left.\mathfrak{Q}_{\mathrm{K}}\right)$ " by " $\left(\mathfrak{Q}_{\mathrm{M}}\right)$ ", "first category" by "measure zero," and "residual subset $R$ " by "subset $R$ such that $E-R$ is of measure zero."

THEOREM 3. The conjunction of $\left(\mathfrak{B}_{K}^{*}\right)$ and $\left(\mathfrak{\Omega}_{R}^{*}\right)$ is equivalent to $(\mathrm{H})$.

Proof. (a) Assume that $(\mathrm{H})$ is true. Then Lemma 1 implies that $\left(\mathfrak{B}_{R}^{*}\right)$ is true, and the truth of $\left(\mathfrak{Q}_{K}^{*}\right)$ follows from Lemma 2 .

(b) Assume that $\left(\mathfrak{B}_{K}^{*}\right)$ and $\left(\mathfrak{Q}_{K}^{*}\right)$ are true. If $(\mathrm{H})$ is false, then, in view of (iii) of $\left(\mathfrak{\Omega}_{R}^{*}\right)$, there exist $\mathfrak{p}$ vertical lines, with $\boldsymbol{\aleph}_{0}<\mathfrak{p}<2^{\aleph_{0}}$, whose union intersects $P-A$ in a set whose orthogonal projection, $S$, on the $y$-axis is of power less than $2 \aleph_{0}$. If $T$ is the intersection of the $y$-axis with the union of the members of $\Phi$, then, by (i) of $\left(\mathfrak{N}_{R}^{*}\right), T$ is a linear set of first category, and $\left(\mathfrak{B}_{K}^{*}\right)$ implies the existence of an $r \in \mathcal{E}$ such that $S \cap T[r]$ is empty. This means that every member of some translation of $\Phi$ intersects each of the aforementioned $\mathfrak{p}$ vertical lines in a point of $A$, which contradicts (ii) of $\left(\mathfrak{Q}_{K}^{*}\right)$. Consequently, $(\mathrm{H})$ is true.

Theorem 4. The conjunction of $\left(\mathfrak{B}_{M}^{*}\right)$ and $\left(\mathfrak{O}_{M}^{*}\right)$ is equivalent to $(\mathrm{H})$.

Proof. In the proof of Theorem 3, replace "( $\left.\mathfrak{B}_{R}^{*}\right)$ " by "( $\left.\mathfrak{B}_{M}^{*}\right)$ ", "( $\left.\mathfrak{R}_{K}^{*}\right)$ " by " $\left(\mathfrak{Q}_{M}^{*}\right)$ ", and "first category" by "measure zero."

TheOREM 5. The conjunction of $(\Omega)$ and $\left(\mathfrak{Q}_{K}^{\prime}\right)$ is equivalent to $(\mathrm{H})$.

Proof. (a) Assume that $(\mathrm{H})$ is true. Then, as we have remarked above, $(\Re)$ is true, and the truth of $\left(\mathfrak{\Omega}_{K}^{\prime}\right)$ follows from Lemma 2.

(b) Assume that $(\Omega)$ and $\left(\mathfrak{Q}_{K}^{\prime}\right)$ are true. If $(H)$ is false, then, in view of (iii) of $\left(\mathfrak{Q}_{K}^{\prime}\right),(\Omega)$ implies that there exist $\mathfrak{p}$ vertical lines, with $\boldsymbol{\aleph}_{0}<p<2 \boldsymbol{N}_{0}$, whose union intersects $\mathbb{P}-A$ in a set whose orthogonal projection, $T$, on the $y$-axis is a linear set of first category. If $S$ is the intersection of the $y$-axis with the union of the members of $\Phi$, then, by (i) of $\left(\mathfrak{Q}_{K}^{\prime}\right), S$ is of power less than $2^{N_{0}}$, and $\left(\mathfrak{B}_{\mathrm{K}}\right)$, which follows from $(\Omega)$ according to Lemma 1 , implies that $\mathcal{E}$ contains a residual subset $R$ with the property that $T \cap S[r]$ is empty for every $r \in R$. This means that, for some $u \in U$, every member of $\Phi[u]$ intersects each of the aforementioned $\mathfrak{p}$ vertical lines in a point of $A$, which contradicts (ii) of $\left(\mathfrak{Q}_{K}^{\prime}\right)$. Consequently, $(\mathrm{H})$ is true.

Theorem 6. The conjunction of $(\mathfrak{M})$ and $\left(\mathfrak{Q}_{M}^{\prime}\right)$ is equivalent to $(\mathrm{H})$.

Proof. In the proof of Theorem 5, replace "( $(\mathbb{R})$ " by "(M)", " $\left(\mathfrak{\Omega}_{K}^{\prime}\right)$ "

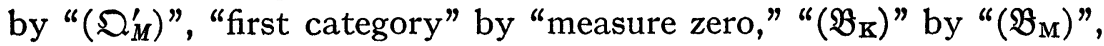
and "residual subset $R$ " by "subset $R$ such that $\varepsilon-R$ is of measure zero." 


\section{REFERENCES}

1. F. Bagemihl, A note on Scheeffer's theorem, Michigan Math. J. vol. 2 (19531954) pp. 149-150.

2. - Some results connected with the continuum hypothesis, Z. Math. Logik Grundlagen Math. vol. 5 (1959).

3. W. Sierpinski, Hypothèse du continu, 2d ed., New York, 1956.

University of Notre DAME 